\title{
Squash vein yellowing virus Detection Using Nested Polymerase Chain Reaction Demonstrates that the Cucurbit Weed Momordica charantia Is a Reservoir Host
}

\author{
Scott Adkins, United States Department of Agriculture-Agricultural Research Service (USDA-ARS), Fort Pierce, \\ FL 34945; Susan E. Webb, University of Florida, Department of Entomology and Nematology, Gainesville 32611; \\ Carlye A. Baker, Florida Department of Agriculture and Consumer Services, Division of Plant Industry, Gainesville \\ 32614; and Chandrasekar S. Kousik, USDA-ARS, Charleston, SC 29414
}

\begin{abstract}
Adkins, S., Webb, S. E., Baker, C. A., and Kousik, C. S. 2008. Squash vein yellowing virus detection using nested polymerase chain reaction demonstrates that the cucurbit weed Momordica charantia is a reservoir host. Plant Dis. 92:1119-1123.

Squash vein yellowing virus (SqVYV) is a recently described ipomovirus from cucurbits in Florida that induces the relatively unusual symptoms in watermelon of plant death and fruit rind necrosis and discoloration, commonly known in Florida as watermelon vine decline. In this report, SqVYV infection of Momordica charantia (Balsam-apple), a common cucurbit weed, collected in 2005 and 2007 from within or adjacent to fields of declining watermelon, is demonstrated through the use of nested polymerase chain reaction (PCR). $M$. charantia plants located in or around fallow watermelon fields between spring and fall 2007 watermelon crops were also infected with SqVYV, indicating that this weed can serve as an oversummering host for this virus. Furthermore, whiteflies were able to acquire SqVYV from infected M. charantia and transmit it to squash and watermelon. Nested PCR was 10 to 1,000 times more sensitive than non-nested PCR for SqVYV detection in several cucurbit hosts, including M. charantia and watermelon. Melothria pendula (creeping cucumber), another common cucurbit weed, was experimentally infected with SqVYV. These results suggest that improved management of $M$. charantia and other cucurbit weeds needs to be incorporated into watermelon vine decline management plans to reduce sources of SqVYV and other cucurbit viruses.
\end{abstract}

Squash vein yellowing virus (SqVYV) was recently isolated from cucurbits in Florida (1). The host range, whitefly transmission, and deduced coat protein (CP) sequence of SqVYV are consistent with it being a novel member of the genus Ipomovirus in the family Potyviridae. SqVYV has been identified in watermelon samples from the past six growing seasons (1; unpublished) from fields exhibiting a vine decline and fruit rind necrosis and discoloration (commonly known in Florida as watermelon vine decline; 12,13). Furthermore, SqVYV was shown in greenhouse experiments to induce watermelon vine decline symptoms, suggesting that it is the cause of this disease (1). SqVYV was known only within Florida until a recent report from Indiana (9).

Corresponding author: S. Adkins

E-mail: scott.adkins@ars.usda.gov

* The $\boldsymbol{e}$-Xtra logo stands for "electronic extra" and indicates that Figures 1 and 2 appear in color in the online edition.

Accepted for publication 17 March 2008.

doi:10.1094/PDIS-92-7-1119

This article is in the public domain and not copyrightable. It may be freely reprinted with customary crediting of the source. The American Phytopathological Society, 2008.
The origin of SqVYV relative to the watermelon vine decline observed in Florida remains unclear. Because SqVYV is so far known only to infect cucurbits (1), it is likely that cucurbit reservoirs exist in nature. Such reservoirs could include cucurbit crops, post-crop volunteer plants, and weeds. The widespread cucurbit weeds Momordica charantia L. (Balsam-apple) and Melothria pendula L. (creeping cucumber) have long been known as reservoir hosts of Papaya ringspot virus type $\mathrm{W}$ (PRSV-W) in Florida (2-4) and other viruses elsewhere $(6,8)$. PRSV-W, an aphidtransmitted potyvirus, is commonly found co-infecting declining watermelons with SqVYV (1). Cucurbit leaf crumple virus (CuLCrV), a begomovirus, was detected recently in the state (16) and, like SqVYV, is transmitted by whiteflies $(5,11)$.

Our original SqVYV isolate, collected in October 2003 in Hillsborough County, FL, was from a squash plant (Cucurbita pepo) with vein yellowing symptoms (1) in a field adjacent to an abandoned citrus grove overgrown with $M$. charantia. Subsequent observations and collections of $M$. charantia were made from within or around fields of declining watermelon in southwest and west-central Florida during the 2005 and 2007 growing seasons (Fig. 1) and between the spring and fall 2007 growing seasons. In one watermelon field in west-central Florida in the fall of 2005, a tree line overgrown with $M$. charantia bordered the entire eastern side of the field (Fig. 1B). No obvious virus-like symptoms were observed on $M$. charantia in and around this field (Fig. 1A). In another watermelon field in southwest Florida in the spring of 2007, M. charantia plants were found growing within the beds containing dead and dying watermelon plants.

In this article, we demonstrate SqVYV infection of $M$. charantia field samples collected during four growing seasons and between two of those growing seasons by using a reverse-transcription polymerase chain reaction (RT-PCR) assay that is more specific for SqVYV and a nested PCR assay that is more sensitive than the original RT-PCR assay we reported for this virus (1). Whitefly acquisition of SqVYV from inoculated $M$. charantia and subsequent transmission to squash and watermelon, with the appearance of typical symptoms, is also shown. The susceptibility of a second common cucurbit weed, $M$. pendula, to infection by SqVYV is demonstrated, suggesting that increased attention to all cucurbit weeds is warranted for management of SqVYV and watermelon vine decline.

\section{MATERIALS AND METHODS}

Virus sources. The original squash isolate of SqVYV was maintained in squash (C. pepo) cvs. Prelude II and Sunray (Seminis Seeds, Oxnard, CA) in a greenhouse by mechanical transmission using $20 \mathrm{mM}$ sodium phosphate buffer $(\mathrm{pH} 7.0)$ containing $0.1 \%(\mathrm{wt} / \mathrm{vol})$ sodium sulfite and $1 \%(\mathrm{wt} / \mathrm{vol})$ Celite. A Florida watermelon isolate of PRSV-W was maintained by the same method in the same hosts.

Plant sources. Seedlings of $M$. charantia and M. pendula were grown in an airconditioned greenhouse under natural lighting with a daytime high temperature of $30^{\circ} \mathrm{C}$. All plants were mechanically inoculated at the five- to six-true-leaf stage with buffer only (mock) or SqVYVinfected squash cv. Prelude II leaves homogenized in the phosphate-sulfite buffer indicated above. Plants were evaluated weekly for symptoms. Systemic SqVYV infection of cucurbit weeds was confirmed by one or more of the following methods: (i) inoculation of squash cv. Prelude II with upper, uninoculated leaves homogenized in phosphate-sulfite buffer followed 
by evaluation of vein yellowing two weeks later; (ii) RT-PCR of the inoculated plants as outlined below; or (iii) whitefly transmission assays as outlined below.

Leaf and stem samples were collected from $24 M$. charantia plants in southwest (Collier and Hendry counties) and westcentral (Polk County) Florida during spring and fall growing seasons in 2005 and 2007 and 13 M. charantia plants between the spring and fall 2007 growing seasons (from fields in which spring watermelons were infected with SqVYV) and frozen for later analysis as outlined below. Another $41 \mathrm{M}$. charantia plants were collected in the fall 2007 growing season from vegetable production areas in southeast (Palm Beach and Broward counties) and a citrus production area in central (Lake County) Florida from which no SqVYV or watermelon vine decline had been reported. Samples from eight additional $M$. charantia plants were collected in summer and fall 2007 in east-central (St. Lucie, Indian River, Martin, and Okeechobee counties) Florida in areas not adjacent to watermelon or other cucurbit production.

Detection of SqVYV by RT-PCR and nested PCR. Our previously reported RTPCR assay, based on the deduced SqVYV $\mathrm{CP}$ gene (1), was modified by relocating the $5^{\prime}$ primer upstream of the $5^{\prime}$ terminus of the CP gene and lengthening the $3^{\prime}$ primer. First-strand cDNA was synthesized from total plant RNA (RNeasy Plant Mini Kit; Qiagen, Valencia, CA) or virion RNA by Moloney murine leukemia virus (MMLV) reverse transcriptase (Promega Corp., Madison, WI) at $45^{\circ} \mathrm{C}$ for $45 \mathrm{~min}$ with standard methods (15) using a previously described degenerate potyvirus $3^{\prime}$ terminus primer (10). The $3^{\prime}$-most $68 \mathrm{nu}$ cleotides of the NIb gene and the entire SqVYV CP gene were amplified by PCR using primers 5'-CCCTCGGAGAACTTG ATATGGAAGCAC-3' and 5'-CGCGTC CTTCCTCTCCAGGCGCTG-3' (complementary to nucleotides 998-1021; GenBank accession no. DQ812125) by 30 cycles with $\mathrm{Taq}$ polymerase (Promega Corp.) at $94^{\circ} \mathrm{C}$ for $30 \mathrm{~s}, 63^{\circ} \mathrm{C}$ for $45 \mathrm{~s}$, and $72^{\circ} \mathrm{C}$ for $45 \mathrm{~s}$. The predicted length of the amplified DNA product was 1,071 bp.

For nested PCR, the initial CP RT-PCR reaction was diluted 1:100 ( $\mathrm{vol} / \mathrm{vol})$ in distilled water and used as template to amplify an internal portion of the CP gene with primers 5'-GCGGGTGAGAAAACC AAGAAGAGACCG-3' and 5'-CTTCCT CATGGTTGGGAGAGCATG-3' (identical to nucleotides 157 to 183 and complementary to nucleotides 724 to 747 , respectively; GenBank accession no. DQ812125). Amplification conditions were as described above but annealing and extension times were reduced to $30 \mathrm{~s}$ each. The predicted length of the amplified DNA product was $591 \mathrm{bp}$.

Initial CP and nested PCR products from squash and watermelon controls, nested PCR products from $M$. charantia, and CP PCR products from $M$. pendula were ligated into pGEM-T (Promega Corp.). These clones were sequenced on an ABI3730XL automated sequencer at the USHRL DNA Sequencing Support Laboratory to confirm the identity of SqVYV.

Sensitivity of nested PCR for SqVYV detection. The sensitivity of the nested PCR assay was directly compared with the non-nested CP RT-PCR assay for SqVYV detection from SqVYV-infected watermelon, $M$. charantia and $M$. pendula total RNA, and SqVYV virion RNA. Mockinoculated squash or watermelon total RNA was used as a control. All RNAs were quantified using a NanoDrop spectrophotometer (NanoDrop Technologies, Wilmington, DE) and diluted to a common concentration of $100 \mathrm{ng} / \mu \mathrm{l}$ permitting use of $250 \mathrm{ng}$ of RNA $(2.5 \mu \mathrm{l}$ of the $100 \mathrm{ng} / \mu \mathrm{l}$ dilution) in each of an initial set of RTPCR reactions with $\mathrm{CP}$ primers. As described above, the products of these initial reactions were diluted 1:100 ( $\mathrm{vol} / \mathrm{vol})$ in distilled water and used for nested PCR. Six 10-fold serial dilutions of the initial $100 \mathrm{ng} / \mu \mathrm{l}$ dilutions of the RNA templates subsequently were prepared to allow testing of $25 \mathrm{ng}, 2.5 \mathrm{ng}, 250 \mathrm{pg}, 25 \mathrm{pg}, 2.5 \mathrm{pg}$, and $250 \mathrm{fg}$ of RNA by these methods.

Detection of PRSV-W and CuLCrV by RT-PCR. First-strand PRSV-W cDNA was synthesized with the above conditions but using random hexamers. A 511-bp portion of the PRSV-W CP gene was amplified by $\mathrm{PCR}$ using primers $5^{\prime}$ GCGGCAGAAGCATATATTGCAAA-3' and 5'-CTCTCATTCTAAGAGGCTCGA ATA-3' (identical to nucleotides 559 to 581 and complementary to nucleotides 1,047 to 1,070 , respectively; GenBank accession no. S89893) with the conditions described above for the SqVYV CP. Previously described degenerate begomovirus primers (PAL1v1978 and PAR1c496) and conditions (14) were used for amplification of CuLCrV sequences. Identities of both PRSV-W and CuLCrV PCR products were confirmed by cloning and sequencing.

Whitefly source, maintenance, and transmission studies. Adult whiteflies (Bemisia tabaci, $\mathrm{B}$ strain $=B$. argentifolii Bellows \& Perring) of mixed ages (newly eclosed to 4 days) were obtained from a colony maintained on cotton (Gossypium hirsutum) and collard (Brassica oleracea var. acephala) as described by Chen and colleagues (7). Source plants were mockinoculated or SqVYV-infected M. charantia (prepared as described above) grown from seed and were tested by nested PCR to confirm SqVYV infection status prior to use in whitefly transmission assays. Test
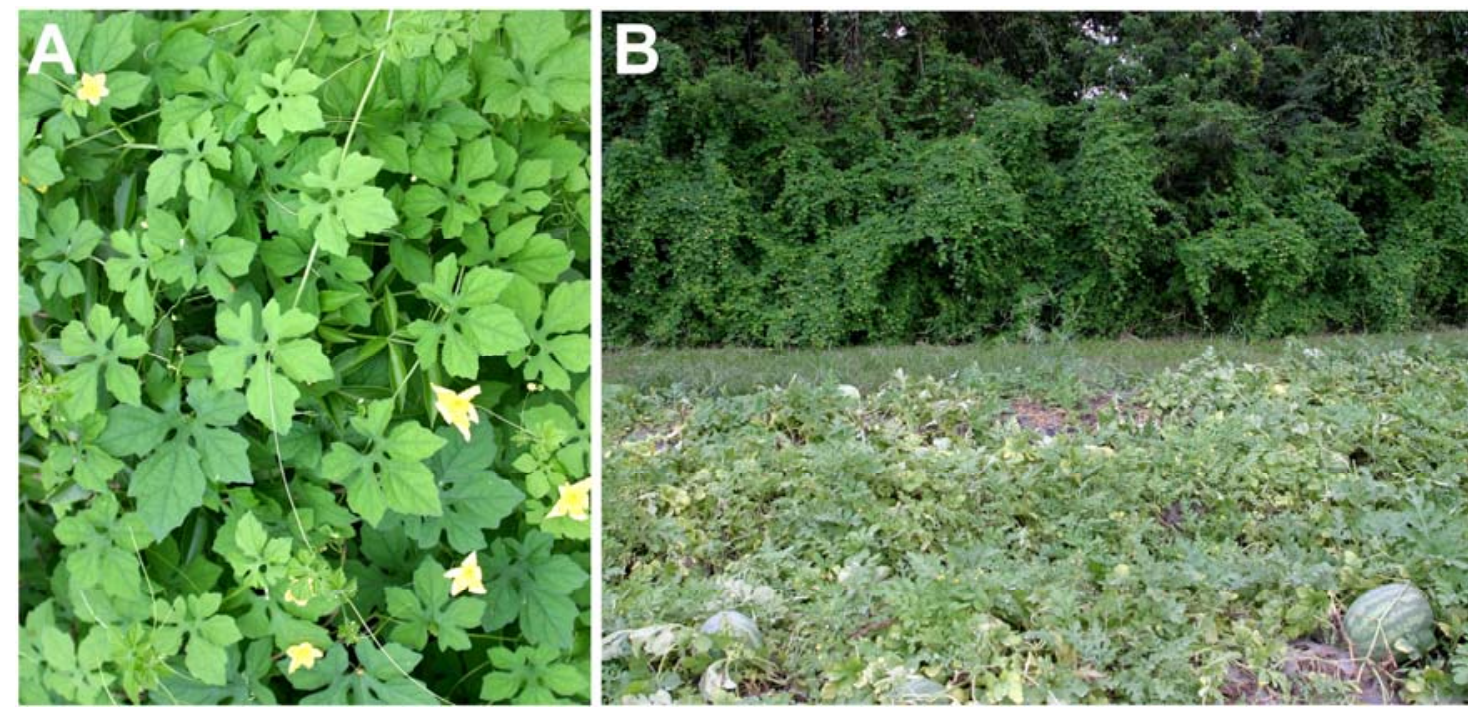

Fig. 1. Momordica charantia with no obvious virus symptoms is commonly found within and adjacent to Florida watermelon fields showing symptoms of vine decline. A, $M$. charantia in west-central Florida in October 2005. B, $M$. charantia growing in a tree line at the edge of watermelon field in early stage of vine decline in west-central Florida in October 2005. Samples from plants in A and B were later determined to be infected with Squash vein yellowing virus. 
plants were squash cvs. Prelude II or Sunray and watermelon cv. Crimson Sweet grown as previously described (1).

Whitefly transmission of SqVYV from infected $M$. charantia. In an initial experiment, approximately 500 whiteflies were released into an insect-proof cage containing three SqVYV-infected $M$. charantia plants at 8 weeks post inoculation. After a 48-h acquisition access period, one 2-week-old watermelon plant was added to the cage. After a 72-h acquisition access period, 11 2-week-old squash plants $(9 \mathrm{cv}$. Sunray and $2 \mathrm{cv}$. Prelude II) were also added to the cage and the $M$. charantia plants were cut off at the crown to induce wilting. Plants were treated 6 days later with pymetrozine (Fulfill; Syngenta, Greensboro, NC) to kill developing whitefly nymphs.

A second mass exposure experiment included a mock-inoculated $M$. charantia plant in a separate cage and two SqVYVinfected $M$. charantia plants at 7 weeks post inoculation caged together. Approximately 500 whiteflies were added to each cage for a 48-h acquisition access period. After this time, three 3-week-old squash plants (cv. Sunray) were added to the cage with the mock-inoculated $M$. charantia

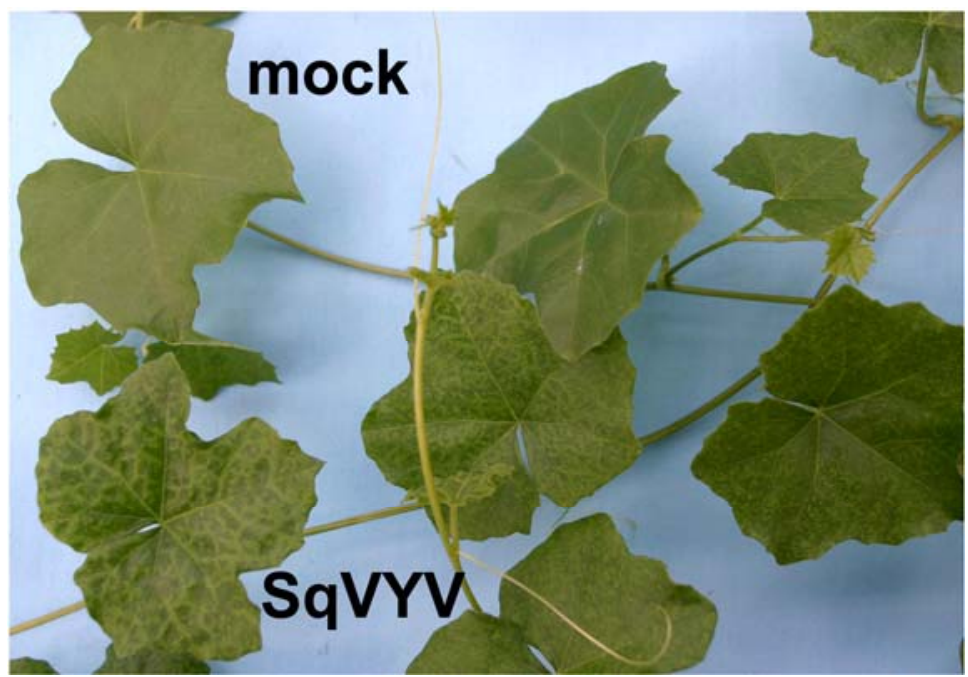

Fig. 2. Melothria pendula is a host for Squash vein yellowing virus (SqVYV). Greenhouse-grown $M$. pendula inoculated and infected with SqVYV shows vein-yellowing symptoms (bottom vine). Mockinoculated plant (top vine) is shown for comparison. plant and nine squash plants were added to the cage with the SqVYV-infected $M$. charantia plants. As before, the $M$. charantia plants were cut off at the soil line so that whiteflies would move to the squash test plants.

Test plants from both experiments were examined for symptoms for 2 weeks following whitefly exposure prior to collecting samples for analysis of transmission. The RT-PCR primers and conditions described above were used to assay for SqVYV.

\section{RESULTS}

Mechanical inoculation of $M$. charantia and $M$. pendula with SqVYV. $M$. charantia plants generally did not show any obvious virus symptoms following inoculation with SqVYV in the greenhouse. Rarely, faint vein-yellowing symptoms were seen in older plants (more than 2 months after inoculation) that resemble those previously reported in squash (1). In contrast, $M$. pendula plants exhibited striking vein-yellowing symptoms following inoculation with SqVYV in the greenhouse (Fig. 2). No symptoms were observed in mock inoculated $M$. charantia or M. pendula plants.

RT-PCR detection of SqVYV. Using total RNA extracted from SqVYV-infected squash (Fig. 3, lanes 5 and 6), products of the expected sizes of 1,071 or $591 \mathrm{bp}$ were observed following RT-PCR amplification with the above-described SqVYV CP or nested primers, respectively. SqVYV was detected by using $\mathrm{CP}$ primers with total RNA from inoculated $M$. pendula plants

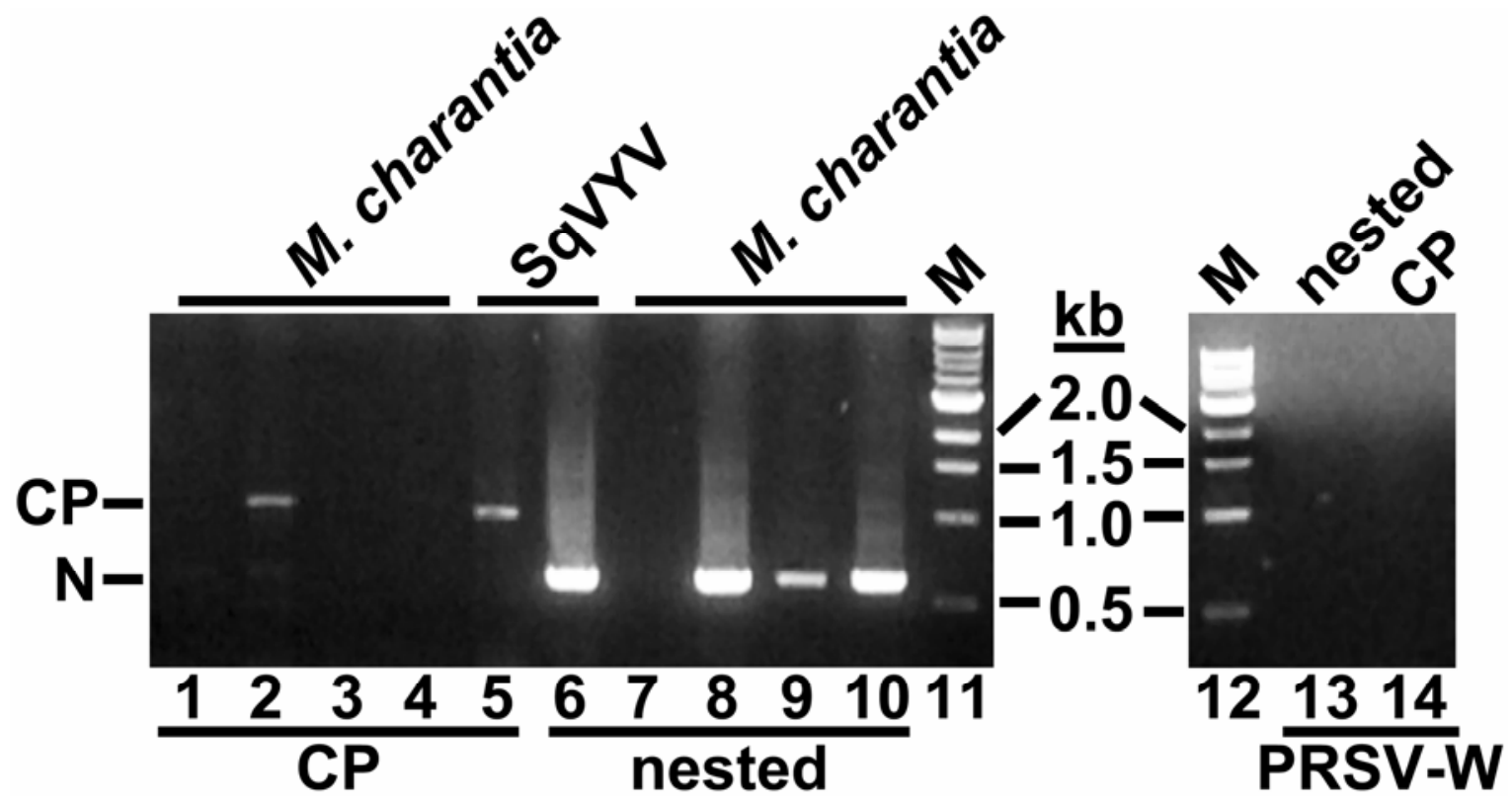

Fig. 3. Detection of Squash vein yellowing virus (SqVYV) by reverse-transcription polymerase chain reaction (RT-PCR) in naturally infected field plants of Momordica charantia is improved by nested PCR. Total RNA was used as template for RT-PCR amplification of the SqVYV coat protein (CP) gene, analyzed by native gel electrophoresis on 1\% agarose gels, and stained with ethidium bromide. Products of initial RT-PCR amplification of CP gene (lanes 1-5, 14) were diluted for subsequent nested PCR (lanes 6-10, 13). RT-PCR products correspond to field samples shown in Figure 1 as follows: Fig. 1A (lanes 2 and 8) and Fig. 1B (lanes 3 and 9). Lanes 5 and 6: Positive-control RT-PCR products from squash plants mechanically inoculated with SqVYV. Lanes 13-14: Negative-control RT-PCR products from squash plants mechanically inoculated with Papaya ringspot virus type W (PRSV-W). Lanes 11 and 12: Markers (M) with size in kilobase (kb) indicated. Relative positions of CP and nested (N) RT-PCR products are indicated to the left of the gel. 
but only by using nested primers with total RNA from inoculated $M$. charantia plants. No amplification products from mockinoculated plants were obtained with either primer set. The SqVYV CP and nested primers and conditions reported here do not amplify a product from PRSV-W RNA (Fig. 3, lanes 13 and 14) unlike our previously described SqVYV CP primers (see Figure 5, lanes 4, 5, and 12 in reference 1).

Detection of SqVYV in $M$. charantia field samples. No obvious virus symptoms were observed in any of 86 field-collected M. charantia from 13 locations in 10 Florida counties, although diffuse chlorotic spots were sometimes seen. SqVYV was detected in $M$. charantia without obvious virus symptoms collected adjacent to or within fields of declining watermelon using CP primers (Fig. 3, lane 2), although nested primers offer increased sensitivity (Fig. 3, lanes 8 to 10). SqVYV was detected in 9 of $24 \mathrm{M}$. charantia plants collected from five agricultural areas experiencing or having experienced watermelon vine decline (southwest and west-central Florida) by using CP (five) or nested (four) primers. Nested PCR products all shared 98 to $99 \%$ nucleotide identity with our original SqVYV isolate (1). Similarly, SqVYV was detected by nested PCR in 7 of $13 M$. charantia plants collected between growing seasons from fields in which spring watermelons were infected with SqVYV. Overall, $43 \%$ of M. charantia samples collected from the five watermelon production locations were positive for SqVYV (Fig. 4). Interestingly, none of these were positive for PRSV-W but a single between-season sample was positive for CuLCrV. SqVYV also was detected in several volunteer watermelon plants collected between growing seasons.

Surprisingly, SqVYV was detected by nested PCR in 4 of $41(10 \%)$ M. charantia plants collected in the fall 2007 growing season from vegetable and citrus production areas in southeast and central Florida.

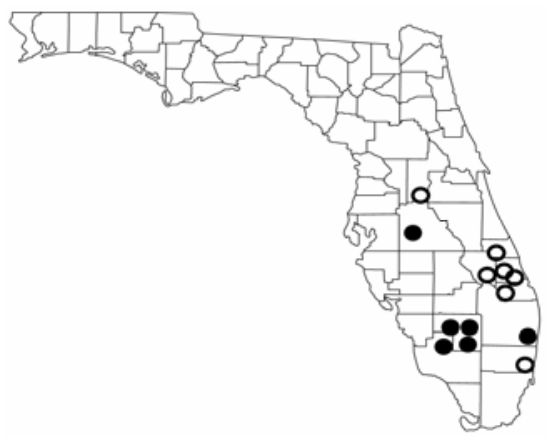

Fig. 4. Distribution of Squash vein yellowing virus in Momordica charantia in peninsular Florida. Map shows Florida counties with 13 locations (indicated by circles) from which 86 $M$. charantia plants were sampled. Filled circles indicate the presence of at least one infected plant as determined by reverse-transcription polymerase chain reaction.
All four samples were from a vegetable production area from which no prior reports of SqVYV or watermelon vine decline have been made. No PRSV-W or $\mathrm{CuLCrV}$ was detected in any of these 41 $M$. charantia plants.

None of the eight samples from the five nonagricultural locations were positive for SqVYV or CuLCrV, although a single sample was positive for PRSV-W, our only finding of this virus in the entire study.

Increased sensitivity of SqVYV detection by nested PCR. SqVYV was detectable from infected watermelon in $25 \mathrm{pg}$ of total RNA by nested PCR but was not detectable in this same amount of total RNA from infected $M$. charantia or infected $M$. pendula. One hundred-fold more total RNA (2.5 ng) was required for SqVYV detection from $M$. charantia and 10-fold more total RNA (250 pg) was required for SqVYV detection from $M$. pendula. The identical trend was observed for watermelon and $M$. pendula with RT-PCR using $\mathrm{CP}$ primers. SqVYV was not detectable from $M$. charantia with RT-PCR using CP primers at the highest amount of total RNA tested. Collectively, these results suggest that SqVYV replicates to the highest titer in watermelon and to the lowest titer in $M$. charantia.

Whitefly transmission. In the first experiment, 2 of 11 squash plants (one cv. Prelude II and one cv. Sunray) and the single watermelon plant began developing symptoms 5 days after exposure to whiteflies fed on SqVYV-infected $M$. charantia plants. In the second experiment, two of nine squash plants began developing symptoms 8 days after exposure to whiteflies fed on SqVYV-infected $M$. charantia plants. Whitefly transmission of SqVYV to these symptomatic plants was confirmed by RT-PCR with CP primers. No symptoms were observed on, and no SqVYV was detected by RT-PCR with CP primers in, any control plants in the cage with the mock-inoculated $M$. charantia plant.

\section{DISCUSSION}

Although lacking obvious virus symptoms (Fig. 1), M. charantia plants collected over four growing seasons from watermelon production areas were often infected with SqVYV as determined by RT-PCR or nested PCR (Fig. 3). M. charantia can be mechanically inoculated and infected with SqVYV in the greenhouse, again usually in the absence of obvious virus symptoms. The increased sensitivity of nested PCR was needed to detect SqVYV in most inoculated and field $M$. charantia plants (Fig. 3), suggesting that SqVYV titer is low within these plants. In spite of the apparent low virus titer, we have shown that whiteflies can acquire SqVYV from infected $M$. charantia plants and transmit it to squash and watermelon target plants, demonstrating that $M$. charantia can serve as a reservoir for SqVYV.
Experimental mechanical inoculations of $M$. pendula show that it also can be infected with SqVYV in the greenhouse as determined by RT-PCR. The detection of SqVYV in $M$. pendula with non-nested RT-PCR suggests that SqVYV reaches a higher titer within $M$. pendula compared with $M$. charantia. $M$. pendula developed striking vein-yellowing symptoms throughout the plants (Fig. 2), unlike M. charantia. Collectively, these results show that $M$. pendula also may be a potential reservoir for SqVYV.

The RT-PCR and nested PCR primers and assays developed and demonstrated in this report offer increased specificity and sensitivity for SqVYV detection compared with our original assay (1). The redesigned $\mathrm{CP}$ primers permit an increase in annealing temperature for RT-PCR to 63 from $55^{\circ} \mathrm{C}$ (1). Increased specificity for SqVYV is demonstrated by the lack of amplification of a product from PRSV-W RNA by RTPCR with the new $\mathrm{CP}$ and nested primers (Fig. 3, lanes 13 and 14), in contrast to our previously described SqVYV CP primers (1). The specificity of RT-PCR with the PRSV-W CP primers reported here was improved by using random hexamers for reverse transcription. Increased sensitivity of SqVYV detection was first observed with the use of nested PCR following initial RTPCR amplification with CP primers from total RNA of field $M$. charantia samples and later, more quantitatively, in an experiment with additional SqVYV-infected hosts. Nested PCR was approximately 10 to 1,000 times more sensitive (depending on the host) than non-nested RT-PCR with CP primers and showed that SqVYV replicated to the highest titer in watermelon and to the lowest titer in $M$. charantia.

$M$. charantia and $M$. pendula are both known as reservoir hosts of PRSV-W in Florida (2-4). Adlerz $(2,3)$ showed that $M$. pendula was an overwintering host for PRSV-W in south and central Florida and noted $(2,4)$ that $M$. charantia and $M$. pendula can survive typical south Florida winters, making them ideal reservoir hosts for PRSV-W and other plant viruses. There are two growing seasons for watermelon in peninsular Florida, one in the fall (late August to early December) and a second in the spring (February to early May). During the course of this study, we commonly observed these weeds during the winter, spring, summer, and fall in Florida; and we have detected SqVYV in spring, summer, and fall $M$. charantia samples. Thus, $M$. charantia, and potentially $M$. pendula, can serve as oversummering hosts for SqVYV in Florida by providing a means to bridge the gap between the spring and fall cucurbit crops. Detection of several SqVYVinfected volunteer watermelon plants collected between the spring and fall 2007 growing seasons suggests that volunteer crop plants are also a potential reservoir and oversummering host for this virus. 
Watermelon plants infected with SqVYV in Florida are also frequently infected with PRSV-W, although SqVYV is sufficient for watermelon vine decline (1). Curiously, none of the $M$. charantia plants collected from areas adjacent to declining watermelon during the 2005 or 2007 growing seasons or near fallow watermelon fields between growing seasons was infected with PRSV-W, whereas 16 of 37 of these plants were infected with SqVYV. PRSVW was, however, detected in one of eight M. charantia plants collected from eastcentral Florida, well outside of cucurbit production areas. Identification of the single $M$. charantia sample collected between growing seasons that was infected with $\mathrm{CuLCrV}$ suggests that this weed will also become an important reservoir and oversummering host for this newly arrived virus.

The geographic distribution of SqVYVinfected $M$. charantia we observed in peninsular Florida in this study (Fig. 4) corresponds well with the major watermelon production areas (southwest and westcentral Florida) and parallels previous reports of watermelon vine decline in Florida $(1,12,13)$. The lone exception was the detection of SqVYV in the southeast Florida vegetable production area from which neither the virus nor watermelon vine decline have been reported previously. Although southeast Florida is not a major watermelon production area, it does contain significant acreages of other cucurbits, including squash and cucumber. Perhaps the aggressive whitefly management program of the grower from whose fields $M$. charantia samples were collected has prevented a significant outbreak of disease due to SqVYV. Regardless, our detection of SqVYV in $M$. charantia in this environment suggests that increased vigilance for this virus is required outside of the currently studied watermelon production areas.
Our data cannot directly address whether SqVYV or CuLCrV moved in the field from $M$. charantia to watermelon or vice versa. However, we have noted (as have others; 2,4$)$ that $M$. charantia can remain year round in south Florida cucurbit production areas, whereas watermelon, squash, and other cucurbit crops do not. We have also definitively shown that whiteflies are capable of transmitting SqVYV from $M$. charantia to squash and watermelon. Collectively, these results suggest that improved management of $M$. charantia, $M$. pendula, and other cucurbit weeds, as well as post-cucurbit crop volunteer plants, needs to be incorporated into management plans for SqVYV and watermelon vine decline and other cucurbit viruses such as $\mathrm{CuLCrV}$.

\section{ACKNOWLEDGMENTS}

We thank C. Vanderspool, M. Gonzalez, N. Miller, J. Smith, R. Lewis, and J. C. Turner for their excellent technical assistance; R. Muchovej, P. Roberts, W. Turechek, and G. McCollum for helping to collect $M$. charantia field samples; and W. Li and W. Turechek for critical reviews of the manuscript.

\section{LITERATURE CITED}

1. Adkins, S., Webb, S. E., Achor, D., Roberts, P. D., and Baker, C. A. 2007. Identification and characterization of a novel whiteflytransmitted member of the family Potyviridae isolated from cucurbits in Florida. Phytopathology 97:145-154.

2. Adlerz, W. C. 1969. Distribution of watermelon mosaic viruses 1 and 2 in Florida. Proc. Fla. State Hortic. Soc. 81:161-165.

3. Adlerz, W. C. 1969. Melothria pendula plants infected with watermelon mosaic virus 1 as a source of inoculum for cucurbits in Collier County, Florida. J. Econ. Entomol. 65:13031306.

4. Adlerz, W. C. 1972. Momordica charantia as a source of watermelon mosaic virus 1 for cucurbit crops in Palm Beach County, Florida. Plant Dis. Rep. 56:563-564.

5. Brown, J. K., Idris, A. M., Alteri, C., and Stenger, D. C. 2002. Emergence of a new cucurbit-infecting begomovirus species capable of forming viable reassortants with related viruses in the Squash leaf curl virus cluster. Phytopathology 92:734-742.

6. Chala, V. H., Harrison, C. W., and Halliwell, R. S. 1987. Identification of two distinct strains of watermelon mosaic virus 2 affecting cucurbits in Texas. Plant Dis. 71:750-752.

7. Chen, J., McAuslane, H. J., Carle, R. B., and Webb, S. E. 2004. Effects of Bemisia argentifolii (Homoptera: Aleyrodidae) infestation and squash silverleaf disorder on zucchini yield and quality. J. Econ. Entomol. 97:2083-2094.

8. Chin, M., Ahmad, M. H., and Tennant, P. 2007. Momordica charantia is a weed host reservoir for Papaya ringspot virus type $\mathrm{P}$ in Jamaica. Plant Dis. 91:1518.

9. Egel, D. S., and Adkins, S. 2007. Squash vein yellowing virus identified in watermelon (Citrullus lanatus) in Indiana. Plant Dis. 91:1056.

10. Gibbs, A., and Mackenzie, A. 1997. A primer pair for amplifying part of the genome of all potyvirids by RT-PCR. J. Virol. Methods 63:916.

11. Guzman, P., Sudarshana, M. R., Seo, Y.-S., Rojas, M. R., Natwick, E., Turini, T., Mayberry, K., and Gilbertson, R. L. 2000. A new bipartite geminivirus (begomovirus) causing leaf curl and crumpling in cucurbits in the Imperial Valley of California. Plant Dis. 84:488.

12. Roberts, P., Muchovej, R. M., Gilreath, P., McAvoy, G., Baker, C. A., and Adkins, S. 2004. Mature vine decline and fruit rot of watermelon. Citrus Veg. Mag. December: 12.

13. Roberts, P., Muchovej, R., Urs, R., Achor, D. Baker, C., Bruton, B., and Adkins, S. 2005. Investigation into a mature watermelon vine decline and fruit rot. (Abstr.) Phytopathology 95:S89.

14. Rojas, M. R., Gilbertson, R. L., Russell, D. R., and Maxwell, D. P. 1993. Use of degenerate primers in the polymerase chain reaction to detect whitefly-transmitted geminiviruses. Plant Dis. 77:340-347.

15. Sambrook, J., and Russell, D. W. 2001. Molecular Cloning: A Laboratory Manual, 3rd ed. Cold Spring Harbor Laboratory, Cold Spring Harbor, NY.

16. Webb, S. E., Akad, F., Nyoike, T. W., Liburd, O. E., and Polston, J. E. 2007. Whiteflytransmitted Cucurbit leaf crumple virus in Florida. Fact Sheet ENY-477. University of Florida, Institute of Food and Agricultural Sciences, Florida Cooperative Extension Service. 